\title{
Detection of G338R FGFR2 mutation in a Vietnamese patient with Crouzon syndrome
}

\author{
ANH LAN THI LUONG ${ }^{1,2^{*}}$, THUONG THI HO ${ }^{3 *}$, HA HOANG $^{3}$, TRUNG QUANG NGUYEN ${ }^{1,2}$, TU CAM HO $^{1}$, \\ PHAN DUC TRAN ${ }^{1,2}$, THUY THI HOANG ${ }^{3}$, NAM TRUNG NGUYEN ${ }^{3,4}$ and HOANG HA CHU ${ }^{3,4}$ \\ ${ }^{1}$ Hanoi Medical University and ${ }^{2}$ Hanoi Medical University Hospital, Kim Lien, Đong Đa; ${ }^{3}$ National Key Laboratory of \\ Gene Technology, Institute of Biotechnology, and ${ }^{4}$ Graduate University of Science and Technology, \\ Vietnam Academy of Science and Technology, Cau Giay, Ha Noi 10000, Vietnam
}

Received September 7, 2018; Accepted December 31, 2018

DOI: $10.3892 /$ br.2019.1181

\begin{abstract}
Crouzon syndrome is a rare autosomal dominant genetic disorder, which causes the premature fusion of the cranial suture. Fibroblast growth factor receptor 2 (FGFR2) mutations are well-known causatives of Crouzon syndrome. The current study aimed to assess the FGFR2 gene associated with Crouzon syndrome in a Vietnamese family of three generations and to characterize their associated clinical features. The family included in the present study underwent complete clinical examination. A patient was clinically examined and presented with typical features of Crouzon syndrome including craniosynostosis, shallow orbits, ocular proptosis and midface hypoplasia. However the patient had normal hands and feet, a normal hearing ability and normal intelligence. Genomic DNA collected from all family members (except from a 16 week-old-foetus) and 200 unrelated control subjects from the same population was extracted from leukocytes obtained from peripheral blood samples. Genomic DNA was extracted from the 16-week-old foetus via the amniotic fluid of the mother. All coding sequences of FGFR2 were amplified via polymerase chain reaction and directly sequenced. A heterozygous FGFR2 missense mutation (c.1012G $>$ C, p.G338R) in exon 10 was identified in the patient with Crouzon but not in other family members, the 16 week-old-foetus or the controls. This mutation was therefore determined to be the causative agent of Crouzon syndrome. In addition, a novel heterozygous silent mutation (c.1164C $>$ T, p.I388I) in exon 11
\end{abstract}

Correspondence to: Dr Hoang Ha Chu, National Key Laboratory of Gene Technology, Institute of Biotechnology, Vietnam Academy of Science and Technology, 18 Hoang Quoc Viet, Cau Giay, Ha Noi 10000, Vietnam

E-mail: chuhoangha@ibt.ac.vn

*Contributed equally

Key words: Crouzon syndrome, fibroblast growth factor receptor 2 mutation, Vietnamese family case, craniosynostosis, molecular analysis of the FGFR2 gene was identified in the patient with Crouzon, his mother and the 16-week-old fetus, but not in other family members. The mutation in exon 10 of FGRF2 was confirmed via restriction-enzyme digestion. The gain of the BsoBI site confirmed the FGFR2 mutation in exon 10 of the patient with Crouzon. This molecular finding may provide useful information to aid clinicians in the diagnosis of Crouzon syndrome and may also aid early prenatal diagnoses.

\section{Introduction}

Crouzon syndrome is a rare genetic disorder that is characterized by a triad of premature craniosynostosis, orbital proptosis and midfacial hypoplasia (1). Crouzon syndrome, which exhibits autosomal dominant inheritance, was first described by Louis Edouard Octave in 1912 (2) and has been identified as one of the most common craniosynostosis syndromes (3). When compared with Crouzon syndrome, Apert syndrome has a broad clinical spectrum, including complex craniofacial involvement, and deformities of the hands, feet and other joints that require multiple surgical procedures to correct (4). In addition to these severe dysmorphologic characteristics, patients with Apert syndrome have been reported to exhibit variable degrees of neurodevelopmental delay, cognitive deficit and mental retardation, while most patients with Crouzon present with normal mental development and intelligence (4). The severity of symptoms in Crouzon syndrome may vary among affected people, even within a family. The worldwide prevalence of Crouzon syndrome is from $1 / 60,000$ to $1 / 1,000$ live births based on several factors, including race, region and ethnicity (5).

Fibroblast growth factors (FGFs) comprise a large group of developmental and physiological signaling molecules. FGFs serve critical roles in the proliferation, migration and particularly in the differentiation of endothelial cells, and serve to promote angiogenesis (6). The specificity of FGFs-FGF receptor interactions and the factors that affect the stability of this complex, have been the focus of many studies $(7,8)$. The human fibroblast growth factor receptor (FGFRs) family is a subfamily of receptor tyrosine kinases (RTKs), which comprise five members: FGFR1, FGFR2, FGFR3, FGFR4 and FGFR5. The common structure of the FGFR comprises 
an intracellular domain with tyrosine kinase activity, an extracellular ligand domain with three immunoglobulin (Ig)-like domains (IgI, IgII and IgIII) and a single transmembrane helix domain $(6,9)$.

Crouzon syndrome formation is closely associated with mutations of the FGFR2 gene $(5,10)$. The FGFR2 gene encodes the FGFR2 protein, which is located on chromosome 10 and consists of $>21$ exons (11). FGFR2 is highly expressed in the foetal brain and in important tissues, including the brain, retina, spinal cord, salivary gland, skin, kidney and uterus of the adult body (12). To date, 60 different mutations of FGFR2 have been linked to Crouzon syndrome formation (11). Additionally, the majority of patients $(80 \%)$ with mutations in exon 8 and 10 are directly associated with this syndrome (5). However, few studies have assessed the influences of other distinct exons that are linked to Crouzon syndrome.

The prenatalidentification of the Crouzon syndrome-causing FGFR2 mutation is crucial for the subsequent pregnancies of affected patients. Prenatal real-time ultrasonographic diagnosis of exophthalmus is possible at the 35 th week of gestation in a foetus of a patient affected with Crouzon syndrome (13). Furthermore, the successful diagnosis of Crouzon syndrome during pregnancy may occur via chorionic villus biopsy using polymerase chain reaction (PCR) by targeting FGFR2, a known mutation found within the pregnant mother (14). However, the diagnosis of Crouzon syndrome remains challenging due to the relatively complicated phenotype and the difficulty and availability of early diagnostic techniques.

The current study assessed the FGFR2 gene associated with Crouzon syndrome in a Vietnamese family and characterized the associated clinical features.

\section{Materials and methods}

Patient recruitment and clinical evaluations. The current study was approved by the Institute of Biotechnology and Hanoi Medical University Hospital (Ha Noi, Vietnam), and informed consent was obtained from all family members prior to blood sample collection. All family members from a Vietnamese family of three generations and the 200 control subjects were recruited at Hanoi Medical University Hospital (Ha Noi, Vietnam) between June and July 2018. The samples were collected from 100 males and 100 unpregnant females between 10-60 years of age at the time of sample collection, and were tested within 1 month from the time of blood collection. A standard ophthalmic test, which included the assessment of visual acuity, refractive error and intraocular/ fluid pressure was performed in patient III-16, who was diagnosed with Crouzon syndrome. Visual acuity was examined using the VISUSCREEN 100/500 acuity chart (Carl Zeiss AG, Oberkochen, Germany). The degree of refractive error in the eye was examined via VISUREF ${ }^{\circledR} 100$ (Carl Zeiss AG) and the intraocular and fluid pressure inside the eye was determined using VISUPLAN 500 (Carl Zeiss AG). Computed tomography (CT) and physical examinations including blood examination, urinalysis, electrocardiogram, chest X-ray, blood biochemistry, blood lipid and blood coagulation tests, were also performed to exclude systemic diseases The biochemical tests were performed using the blood of patient III-16 using a Biochemistry Analyzer Machine with biochemistry test kit (cat. no. MSLBA28; MSL Electronic Technology (Shenzhen) Co., Ltd., Shenzhen, China). This was utilized to measure sodium, potassium, chloride, bicarbonate, blood urea nitrogen, magnesium, creatinine, glucose and calcium. An ultrasound examination was used to assess a 16-week-old fetus (III-18) of mother (II-16).

Sample collection. The peripheral blood leucocytes of all family members (excluding the 16-week-old fetus) were separated from whole blood using a Histopaque-1077 kit (Sigma-Aldrich; Merck KGaA, Darmstadt, Germany; cat. no. 10771) according to manufacturer's protocol. Genomic DNA samples were then extracted from the peripheral blood leucocytes of all family members (excluding the 16-week-old foetus) using the Qiagen QIAamp DNA Mini kit (Qiagen Inc., Valencia, CA, USA) according to manufacturer's protocol. A foetal genomic DNA sample was carefully extracted from the amniotic fluid of the mother using the QIAamp Circulating Nucleic Acid kit (Qiagen Inc.) according to the manufacturer's protocol. In addition, DNA samples collected from 200 patients in the same population that did not present with diagnostic features of Crouzon syndrome were used as controls. DNA concentration and purity was measured using a NanoDrop ${ }^{\mathrm{TM}}$ ND-1000 spectrophotometer (Thermo Fisher Scientific, Inc., Waltham, MA, USA). Genomic DNA samples were preserved at $-20^{\circ} \mathrm{C}$ prior to use.

Mutation detection and analysis. Nearly all the FGRF2 gene coding sequences were amplified in family members and controls using PCR with Taq DNA polymerase (Thermo Fisher Scientific, Inc.) and specific primers sequences previously designed by Kan et al (15) (listed in Table I) . A different set of primers were utilized for the amplification of exon 10 and part of the intron between exon 10 and 11 for uncomplicated confirmation by restriction enzyme. These primer sequences were as follows: forward, 5'-CCTCCACAATCATTCCTGTGTC-3' and reverse, 5'-TATCGCAACATGCAGCAAGC-3' (product size 733 bp). DNA (100 ng) in a $50 \mu \mathrm{l}$ reaction was amplified. All reagents used for PCR were purchased from Thermo Fisher Scientific, Inc. The amplification included a single 5 min step at $94^{\circ} \mathrm{C}$ followed by 40 cycles of $94^{\circ} \mathrm{C}$ for $45 \mathrm{sec}$, annealing [at temperatures stated by Kan et al (15), and listed in Table I] for $45 \mathrm{sec}$ and $72^{\circ} \mathrm{C}$ for $45 \mathrm{sec}$ followed by a final 10 min step at $72^{\circ} \mathrm{C}$. Products were separated using $1 \%$ agarose gel electrophoresis and stained with ethidium bromide. DNA products then were purified using a QIAquick PCR Purification kit (Qiagen Inc.) and sequenced in each direction using an ABI3100 Genetic Analyzer (Thermo Fisher Scientific, Inc.). The sequencing results were analysed using SeqMan (version 2.3; Technelysium Pty, Ltd., South Brisbane, QLD, Australia) and compared against reference sequences obtained from the ENSEMBL Human Genome Browser (http://asia.ensembl.org/Homo_sapiens/Info/Index) with the code of ENST00000358487. The mutation in exon 10 of the FGFR2 gene was confirmed via BsoBI restriction-enzyme digestion with a recognition site of $\mathrm{C} \downarrow$ YCGR $\uparrow \mathrm{G}$. The location of exon 10 in the FGFR2 gene where the heterozygous missense mutation occurred was from 1010 to 1015 with the change of sequence from CTGGGG to CTCGGG. The DNA from a patient containing this heterozygous mutation was 
Table I. Primers for amplification of FGFR2.

Primer sequences

\begin{tabular}{|c|c|c|c|c|}
\hline Target & Forward & Reverse & $\begin{array}{l}\text { Fragment } \\
\text { size (bp) }\end{array}$ & $\begin{array}{l}\text { Temperature } \\
\left({ }^{\circ} \mathrm{C}\right) \text { annealing }\end{array}$ \\
\hline Exon 2 & TCCCTGACTCGCCAATCTCTTTC & TGCCCCCAGACAAATCCCAAAAC & 341 & 55 \\
\hline Exon 3 & CACTGACCTTTGTTGGACGTTC & GAGAAGAGAGAGCATAGTGCTGG & 380 & 64 \\
\hline Exon 4 & TGGAGAAGGTCTCAGTTGTAGAT & AGACAGGTGACAGGCAGAACT & 232 & 55 \\
\hline Exon 5 & CAAAGCGAAATGATCTTACCTG & AGAAATGTGATGTTCTGAAAGC & 291 & 62 \\
\hline Exon 6 & GCTAGGATTGTTAAATAACCGCC & AAACGAGTCAAGCAAGAATGGG & 226 & 62 \\
\hline Exon $7\left(5^{\prime}\right)$ & TGAGTTTGCCTCTCCTCGTGTG & CCTTCTACAGTTGCCCTGTTGG & 390 & 62 \\
\hline Exon 7(3') & GATGTGCTGTAGCAGACCTTTGG & ATCATCACAGGCAAAACCTGGG & 360 & 62 \\
\hline Exon 8 & GGTCTCTCATTCTCCCATCCC & CCAACAGGAAATCAAAGAACC & 325 & 62 \\
\hline Exon 9 & AATGCTAAGACCTTCCTGGTTGG & CAGTCTCCCAAAGCACCAAGTC & 284 & 55 \\
\hline $\begin{array}{l}\text { Exon } 10 \text { and } \\
\text { part of intron }\end{array}$ & ССТCСАCAATCATTCCTGTGTC & TATCGCAACATGCAGCAAGC & 733 & 53 \\
\hline Exon 11 & TGCGTCAGTCTGGTGTGCTAAC & AGGACAAGATCCACAAGCTGGC & 341 & 64 \\
\hline Exon 12 & TGACTTCCAGCCTTCTCAGATG & AGTCTCCATCCTGGGACATGG & 252 & 64 \\
\hline Exon 13 & CCCCATCACCAGATGCTATGTG & TTGATAAGACTCTCCACCCAGCC & 221 & 55 \\
\hline Exon 14 & TAGCTGCCCATGAGTTAGAGG & ATCTGGAAGCCCAGCCATTTC & 250 & 62 \\
\hline Exon 15 & TGTTTTGCTGAATTGCCCAAG & TCCACCCAGCCAAGTAGAATG & 294 & 55 \\
\hline Exon 16 & CTGGCGGTGTTTTGAAATTAG & СCTTTCTTCCTGGAACATTCTG & 242 & 60 \\
\hline Exon 17 & AGCCCTATTGAGCCTGCTAAG & CCAGGAAAAAGCCAGAGAAAAG & 177 & 62 \\
\hline Exon 18 & GGTTTTGGCAACGTGGATGGG & GGTATTACTGGTGTGGCAAGTCC & 250 & 60 \\
\hline Exon 19 & ACACCACGTCCCCATATTGCC & CTCACAAGACAACCAAGGACAAG & 243 & 60 \\
\hline Exon 20 & TCTGCCAAAATTGTTGTTTCTAGT & GGTCTGGAACTCCTGACCTCA & 208 & 60 \\
\hline Exon 21 & TCCCACGTCCAATACCCACATC & TACTGTTCGAGAGGTTGGCTGAG & 196 & 62 \\
\hline Exon 22 & CGTCCAATACCCACATCTCAAG & TTCCCAGTGCTGTCCTGTTTGG & 363 & 60 \\
\hline
\end{tabular}

gain of the BsoBI site in exon 10 of the FGFR2 gene. Purified PCR products containing the FGFR2 exon 10 of patient III-16, other family members and controls were digested with BsoBI. Digestion products were separated via $2 \%$ agarose gel electrophoresis and stained with ethidium bromide.

The possible impact of the G338R exon 10 mutation on the structure and function of FGFR2 was predicted using PolyPhen-2 (Polymorphism Phenotyping v2; http://genetics. bwh.harvard.edu/pph2/dbsearch.shtml). The effect of the G338R exon 10 mutation on FGFR2 function, based on sequence homology and the physical properties of amino acids, was predicted by sorting intolerant from tolerant (SIFT, http://sift.bii.a-star.edu.sg/). Substitutions with a score $<0.05$ are predicted to affect protein function.

\section{Results}

Clinical presentation. Personal and family histories were reviewed for each member of the three-generation Vietnamese family (Fig. 1A). Systemic diseases were excluded upon examination. Based on the results of all blood biochemical tests, blood lipid, blood coagulation, urinalysis, electrocardiogram and X-rays all appeared normal/within the normal range. All results of these tests were within the normal range (data not shown). Patient III-16 was a 10-year-old boy of two healthy parents. He was born following a normal pregnancy and was diagnosed with Crouzon syndrome at 6 years of age due to his prominent eyes. Clinical examination of this patient indicated that he had shallow orbits and ocular proptosis, mid-face hypoplasia and craniosynostosis (Fig. 1B). He also exhibited bulging eyes, leading to a reduction in his vision, a prominent nasal bridge, an underdeveloped upper jaw and a protruding lower jaw. In addition, his teeth were overcrowded and he underwent surgery to resolve this. Furthermore, his skull appeared 'too tall' and overly flat from the middle part of the face upward. No abnormalities were detected in his hands and feet. His hearing and his physical examination results were normal. He presented with no mental retardation and had been in primary school at the appropriate age. The CT scan confirmed shallow orbits and exotropia in each eye of the patient (Fig. 1C). Ultrasound examination of a 16-week-old fetus revealed a normal appearance (data not shown). Both parents (II-11 and II-16) and daughter (III-17) exhibited normal visual acuity, with unremarkable eye examinations and all family members had no known history of learning difficulties or genetic problems.

Mutation screening. All coding sequences of the FGRF2 gene were amplified using PCR and directly sequenced to identify the mutations in all family members and controls. Sequencing results indicated that there were no mutations identified in other exons of the FGFR2 gene, excluding exon 10 and 11 (data not shown).

Patient III-16 carried a heterozygous missense mutation (c.1012G>C; p.G338R) in exon 10 of the FGFR2 gene (Fig. 2A). This mutation was observed in patient III-16 only and was not 


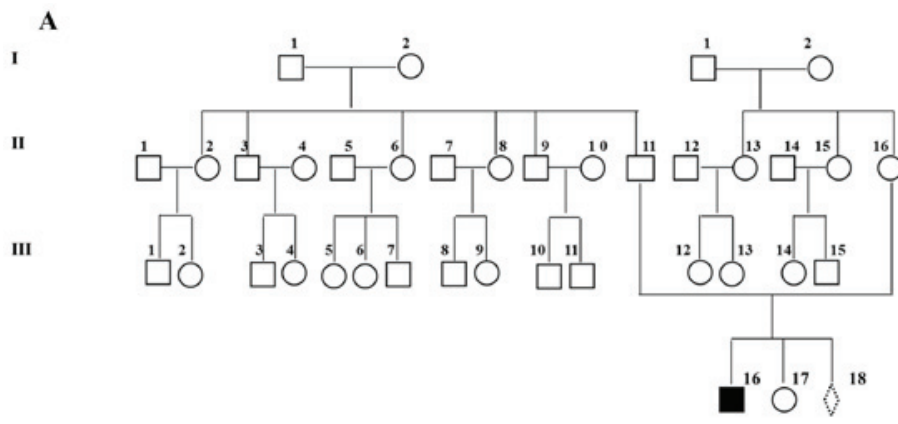

B

\section{III-16}

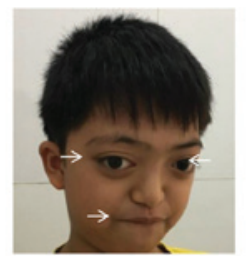

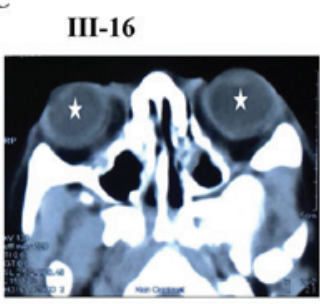

Figure 1. Clinical manifestations of patient III-16. (A) Personal and family history of the Vietnamese family presenting with Crouzon syndrome. Squares denote males, circles denote females and rhombus denotes the 16-week-old fetus. (B) Patient III-16 presented with micrognathia of the upper jaw (white arrows), orbit and ocular proptosis (white arrows), midface hypoplasia and craniosynostosis. (C) A CT scan of patient III-16 revealed shallow orbits and exotropia in each eye (white stars).

A

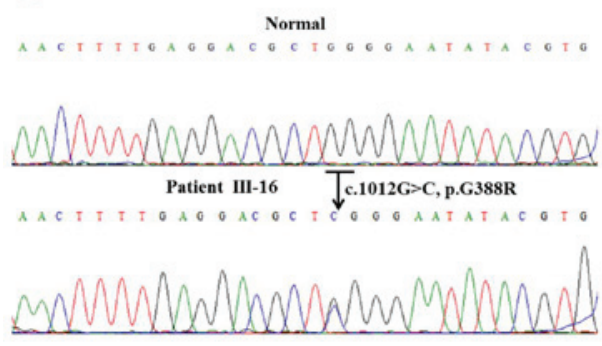

B
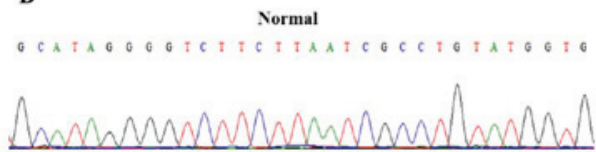

Normal (II-16, III-18) and patient III-16 \.11640T, p.13881

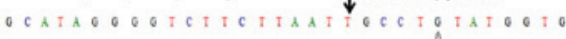

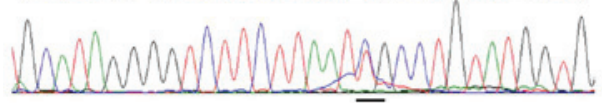

C

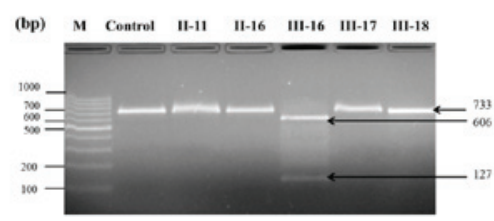

D

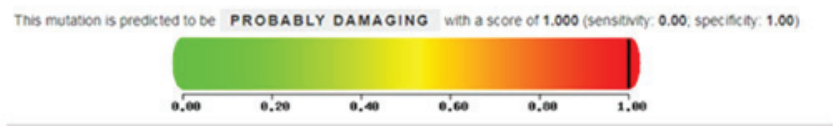

$\mathbf{E}$

Substitution at pos 338 from 6 to $R$ is predicted to Afrect photezw function with o score of 0.01 . Sequences represented ot this position: 29

Figure 2. Identification of two heterozygous missense FGFR2 mutations in exon 10 and exon 11. (A) The DNA-sequence electropherogram of a normal individual compared with that of patient III-16. A heterozygous missense mutation (c.1012G>C; p.G388R) in exon 10 of the FGFR2 gene was identified. (B) The DNA-sequence electropherogram of normal individuals compared with unaffected family members and patient III-16. A heterozygous silent FGFR2 mutation (c.1164C >T; p.I388I) in exon 11 was identified in II-16, III-18 and III-16. (C) The FGFR2 mutation in exon 10 was confirmed using BsoBI. Only exon 10 of patient III-16 was completely digested by BsoBI. (D) The possible impact of the G338R exon 10 mutation on the structure and function of FGFR2 was predicted using PolyPhen-2. The mutation was predicted to be 'probably damaging' with a score of 1.000 . (E) The effect of the G338R exon 10 mutation on FGFR2 function based on sequence homology and the physical properties of amino acids was predicted by SIFT. Substitutions with a score $<0.05$ are predicted to affect protein function.

present in the unaffected family members, the 16 -week-old foetus or the unrelated controls. The mutation was located at the coding region of Ig-like domain 3 and caused the replacement of glycine by arginine at amino acid position 338. This region is a very highly conserved segment of the FGFR2 gene.

Patient III-16, his mother II-16 and the 16-week-old foetus III-18 carried a heterozygous silent mutation (c.1164C $>$ T;
p.I388I) in exon 11 of the FGFR2 gene (Fig. 2B). However, other unaffected family members and unrelated controls did not carry any heterozygous silent mutations in this exon.

The mutation in exon 10 of the FGFR2 gene was confirmed by restriction enzyme digestion. The results revealed that a mutation was present in exon 10 of the FGFR2 gene of patient III-16 due to the gain of the BsoBI site, which resulted in the 
appearance of two bands in the agarose gel (606 bp and $127 \mathrm{bp}$; Fig 2C). In contrast, no BsoBI site was exhibited in the other family members and controls (data not shown), producing only one band in the agarose gel (733 bp; Fig. 2C).

A possible impact of the G388R exon 10 mutation on the structure and function of FGFR 2 by PolyPhen- 2 was predicted. The G338 mutation was predicted to be 'probably damaging' to the structure and function of FGFR2, with a score of 1.000 (Fig. 2D). Furthermore, the effect of the G338R exon 10 mutation on FGFR2 function was predicted by SIFT based on sequence homology and the physical properties of amino acids. The G338R mutation was predicted to affect FGFR2 function with score of 0.01 (Fig. 2E).

\section{Discussion}

Crouzon syndrome is an autosomal dominant disorder with variable expressivity that is characterized by craniosynostosis, shallow orbits, ocular proptosis and midface hypoplasia (16). In the current study, the patient III-16 was clinically examined and presented with typical features of Crouzon syndrome, but not Apert syndrome. The current study excluded the diagnosis of Apert syndrome, as the patient exhibited no abnormalities in the hands and feet, normal mental development and normal intelligence. FGFR2 mutations are a well-known cause of Crouzon and Apert syndrome, but each are caused by a different mutation in FGFR2. Two heterozygous gain-of-function substitutions (Ser252Trp and Pro253Arg) in exon IIIa of FGFR 2 are responsible for $>98 \%$ of Apert syndrome cases (17). To date, the G338R mutation in FGFR2 has not been detected in patients with Apert syndrome.

The current study identified a heterozygous missense mutation (c.1012G>C; p.G338R) in a Vietnamese family with autosomal dominant Crouzon syndrome. This mutation had been previously detected in British and Chinese cases $(15,18)$ but not in the Vietnamese population. In comparison to the clinical manifestations of patients in a previous study (18), patient III-16 exhibited similar symptoms, including shallow orbits and ocular proptosis, accompanied with craniosynostosis and mid-face hypoplasia. However, some clinical manifestations of patient III-16 were different, including normal hands, normal feet and a normal hearing ability. Furthermore, his teeth were overcrowded, which required surgery to resolve. Patient III-16, also did not present with mental retardation, had a normal intelligence and did not exhibit any other abnormality following physical examination. Therefore, the severity of Crouzon syndrome signs and symptoms can vary among affected patients, even with the same FGFR2 mutation.

Previous studies have indicated that the most common genetic mutation of FGFR2 is localized to the third Ig-like domain coded by exons IIIa (exon 8) and IIIc (exon 10) $(15,19,20)$. The G338R mutation identified in the family included the present study was localized to exon IIIc. Most of the mutations in exon 10 of FGFR2 results in a gain or loss of cysteine residues associated with Crouzon syndrome $(5,11,15)$. Furthermore, the cysteine residues of each site in exon 10 of the FGFR2 gene form the disulfide bond in the third Ig-like domain, which controls FGFR2 receptor activity to the FGFR2 protein $(11,21,22)$. Therefore, mutations may lead to a conformational change in the extracellular Ig-III loop or may disrupt the intra-Ig domain disulfide bond, resulting in changes to FGF signaling and the activation or down regulation of FGFR2 $(11,21,22)$. Fan et al (18) utilized a three-dimensional structural model to assess the position of the missense mutation, G338R. The results of the aforementioned study revealed the presence of a hydrogen bond between Tyr328 and Arg330 in mutant-type FGFR2, but not in wild-type FGFR2. This bond is situated near the Gly338 amino acid residue, which may reduce the stability of the FGFR2 protein (18). Furthermore, the expression of two osteoblast specific genes, osteocalcin and alkaline phosphatase, were significantly increased in the orbital bone of patients compared to normal individual (18).

The current study identified a heterozygous silent mutation (c.1164C>T) in exon 11 of the FGFR2 gene in patient III-16, II-16 and III-18 but not in II-11, III-17 and other family members. However, this mutation did not lead to changes in amino acids and may therefore be a single nucleotide polymorphism. Furthermore, different silent mutations in exon 11 and others of the FGFR2 gene were identified in previous studies $(15,23)$.

In summary, a heterozygous missense mutation in exon 10 of the FGFR2 gene associated with Crouzon syndrome and a novel heterozygous silent mutation in exon 11 of the FGFR2 gene were identified in a Vietnamese family of three generations. These results may not only expand the reported mutation spectrum of $F G F R 2$, but may also provide useful information to aid clinicians to confirm the diagnosis of Crouzon syndrome. Furthermore, this molecular analysis may also have a considerable impact on prenatal diagnoses.

\section{Acknowledgements}

The authors would like to thank the patient and family members for their participation.

\section{Funding}

The present study was supported by the National Key Laboratory of Gene Technology, Institute of Biotechnology (Vietnam Academy of Science and Technology; grant no. NV03-PTNTD2017) and Hanoi Medical University Hospital (Vietnam) grant no. KHCN-33.05.01/11-15.

\section{Availability of data and materials}

The datasets used and/or analysed during the current study are available from the corresponding author on reasonable request.

\section{Authors' contributions}

TTH, NTN and Ha HC designed the current study. AL, TQN, TCH and PDC performed clinical examinations. HH extracted DNA. TTH and HH amplified all exons of FGFR2 using PCR and purified the PCR products. Ha $\mathrm{H}$ performed sequencing. TTH analysed the sequencing results, and Thuy TH performed restriction enzyme digestion. TTH and AL wrote the manuscript. NTN and HHC revised the manuscript. HHC is the corresponding author and holds all the responsibilities associated with this manuscript. All authors have read and approved the final manuscript. 


\section{Ethics approval and consent to participate}

All procedures were performed in a bioassay laboratory and approved by the local ethics committee of Hanoi Medical University Hospital and Institute of biotechnology, Vietnam Academy of Science and Technology (Ha Noi, Vietnam).

\section{Patient consent for publication}

The patient and parents provided written informed consent for the publication of any associated data and accompanying images.

\section{Competing interests}

The authors declare that they have no competing interests.

\section{References}

1. Giordano BP, Tuli SS, Ryan SF, Stern M and Tuli SY: Crouzon Syndrome: Visual Diagnosis. J Pediatr Health Care 30: 270-273, 2016.

2. Mitulla B, Hinkel GK and Lorenz P: Crouzon syndrome (Mc K 12350). Kinderarztl Prax 59: 278-280, 1991 (In German).

3. Liu J, Kwon TG, Nam HK and Hatch NE: Craniosynostosisassociated Fgfr2(C342Y) mutant bone marrow stromal cells exhibit cell autonomous abnormalities in osteoblast differentiation and bone formation. Biomed Res Int 2013: 292506, 2013.

4. Raposo-Amaral CE, Neto JG, Denadai R, Raposo-Amaral CM and Raposo-Amaral CA: Patient-reported quality of life in highest-functioning Apert and Crouzon syndromes: A comparative study. Plast Reconstr Surg 133: 182e-191e, 2014.

5. Lin Y, Gao H, Ai S, Eswarakumar JVP, Zhu Y, Chen C, Li T, Liu B, Jiang H, Liu Y, et al: FGFR2 mutations and associated clinical observations in two Chinese patients with Crouzon syndrome. Mol Med Rep 16: 5841-5846, 2017.

6. Korc $\mathrm{M}$ and Friesel RE: The role of fibroblast growth factors in tumor growth. Curr Cancer Drug Targets 9: 639-651, 2009.

7. Chae YK, Ranganath K, Hammerman PS, Vaklavas C, Mohindra N, Kalyan A, Matsangou M, Costa R, Carneiro B, Villaflor VM, et al: Inhibition of the fibroblast growth factor receptor (FGFR) pathway: The current landscape and barriers to clinical application. Oncotarget 8: 16052-16074, 2017.

8. Touat M, Ileana E, Postel-Vinay S, André F and Soria JC: Targeting FGFR signaling in cancer. Clin Cancer Res 21: 2684-2694, 2015.

9. Belov AA and Mohammadi M: Molecular mechanisms of fibroblast growth factor signaling in physiology and pathology. Cold Spring Harb Perspect Biol 5: pii: a015958, 2013.

10. Jabs EW, Li X, Scott AF, Meyers G, Chen W, Eccles M, Mao JI, Charnas LR, Jackson CE and Jaye M: Jackson-Weiss and Crouzon syndromes are allelic with mutations in fibroblast growth factor receptor 2. Nat Genet 8: 275-279, 1994.
11. Li ZL, Chen X, Zhuang WJ, Zhao W, Liu YN, Zhang FX, Ha RS, Wu JH, Zhao C and Sheng XL: FGFR2 mutation in a Chinese family with unusual Crouzon syndrome. Int J Ophthalmol 9: 1403-1408, 2016.

12. Katoh M: (2008). FGFR 2 (fibroblast growth factor receptor 2). Atlas of Genetics and Cytogenetics in Oncology and Haematology. http://atlasgeneticsoncology.org/Genes/GC_ FGFR2.html\#LINKS. Accessed 18 December 2018.

13. Menashe Y, Ben Baruch G, Rabinovitch O, Shalev Y, Katzenlson MB and Shalev E: Exophthalmus - prenatal ultrasonic features for diagnosis of Crouzon syndrome. Prenat Diagn 9: 805-808, 1989.

14. Schwartz M, Kreiborg S and Skovby F: First-trimester prenatal diagnosis of Crouzon syndrome. Prenat Diagn 16: 155-158, 1996.

15. Kan SH, Elanko N, Johnson D, Cornejo-Roldan L, Cook J, Reich EW, Tomkins S, Verloes A, Twigg SR, Rannan-Eliya S, et al: Genomic screening of fibroblast growth-factor receptor 2 reveals a wide spectrum of mutations in patients with syndromic craniosynostosis. Am J Hum Genet 70: 472-486, 2002.

16. Reardon W, Winter RM, Rutland P, Pulleyn LJ, Jones BM and Malcolm S: Mutations in the fibroblast growth factor receptor 2 gene cause Crouzon syndrome. Nat Genet 8: 98-103, 1994.

17. Bochukova EG, Roscioli T, Hedges DJ, Taylor IB, Johnson D, David DJ, Deininger PL and Wilkie AO: Rare mutations of FGFR2 causing apert syndrome: Identification of the first partial gene deletion, and an Alu element insertion from a new subfamily. Hum Mutat 30: 204-211, 2009.

18. Fan J, Li Y, Jia R and Fan X: An inherited FGFR2 mutation increased osteogenesis gene expression and result in Crouzon syndrome. BMC Med Genet 19: 91, 2018.

19. Tartaglia M, Valeri S, Velardi F, Di Rocco C and Battaglia PA: Trp290Cys mutation in exon IIIa of the fibroblast growth factor receptor 2 (FGFR2) gene is associated with Pfeiffer syndrome. Hum Genet 99: 602-606, 1997.

20. Ke R, Yang X, Tianyi C, Ge M, Lei J and Mu X: The C342R mutation in FGFR2 causes Crouzon syndrome with elbow deformity. J Craniofac Surg 26: 584-586, 2015.

21. Lapunzina P, Fernández A, Sánchez Romero JM, Delicado A, Sáenz de Pipaon M, López Pajares I and Molano J: A novel insertion in the FGFR2 gene in a patient with Crouzon phenotype and sacrococcygeal tail. Birth Defects Res A Clin Mol Teratol 73: 61-64, 2005.

22. Piccione M, Antona V, Niceta M, Fabiano C, Martines M, Bianchi A and Corsello G: Q289P mutation in the FGFR2 gene: First report in a patient with type 1 Pfeiffer syndrome. Eur J Pediatr 168: 1135-1139, 2009.

23. Li X, Park WJ, Pyeritz RE and Jabs EW: Effect on splicing of a silent FGFR2 mutation in Crouzon syndrome. Nat Genet 9: 232-233, 1995.
This work is licensed under a Creative Commons Attribution-NonCommercial-NoDerivatives 4.0 International (CC BY-NC-ND 4.0) License. 